\title{
Role of Intraoperative Transesophageal Echocardiography in Evaluation of Fontan Conduit for Thrombosis
}

${ }^{1}$ Sambhunath Das, ${ }^{2}$ Suruchi Ladha, ${ }^{3}$ Balram Airan

\begin{abstract}
The formation of thrombus after Fontan operation is a lifethreatening situation. The clinical manifestations by the patient may not reflect the true diagnosis of thrombosis. Transesophageal echocardiographic evaluation is an option to delineate the vena cava anastomosis and the Fontan conduit in different views. We used transesophageal echocardiography (TEE) to detect the thrombus site prior to surgery and guided for a successful removal of thrombus. The successful surgical revision of the anastomosis was confirmed by TEE.
\end{abstract}

Keywords: Fontan operation, Single ventricle, Thrombosis, Transesophageal echocardiography.

How to cite this article: Das S, Ladha S, Airan B. Role of Intraoperative Transesophageal Echocardiography in Evaluation of Fontan Conduit for Thrombosis. J Perioper Echocardiogr 2015;3(2):55-57.

\section{Source of support: Nil}

Conflict of interest: None

\section{INTRODUCTION}

The Fontan circulation is a low-flow, low-pressure circulation in which areas of stagnation resulting from anastomosis site stricture can create a favorable environment for thrombus formation. ${ }^{1}$ Reliable detection of thrombi by transesophageal echocardiography (TEE) within the Fontan conduit is one of the unique challenges faced by an anesthesiologist caring for a patient with Fontan circulation in the perioperative period..$^{2,3}$

\section{CASE REPORT}

We report a case of 12 years old child having cyanotic congenital heart disease with complete atrioventricular septal defect (AVSD), double-outlet right ventricle (DORV), hypoplastic left ventricle and pulmonary stenosis. The patient underwent a staged palliation for

\footnotetext{
${ }^{1}$ Additional Professor, ${ }^{2}$ Senior Resident, ${ }^{3}$ Professor

1,2Department of Cardiac Anesthesia, All India Institute of Medical Sciences, New Delhi, India

${ }^{3}$ Department of Cardiothoracic and Vascular Surgery, All India Institute of Medical Sciences, New Delhi, India

Corresponding Author: Sambhunath Das, Additional Professor, Department of Cardiac Anesthesia, All India Institute of Medical Sciences, New Delhi, India, Phone: 26593423 e-mail: sambhunathds833@gmail.com
}

univentricular physiology in which a bidirectional Glenn was performed at 2 years of age and intraextracardiac total cavopulmonary connection (TCPC) was performed at 10 years of age. An $18 \mathrm{~mm}$ polytetrafluoroethylene graft (Gore-Tex graft, WL Gore and Assoc Newark, DE) was anastomosed to opening of inferior vena cava and the right atrium was closed over the conduit covering $2 / 3$ rd intracardiac and remaining $1 / 3$ rd extracardiac. A fenestrated Fontan with augmentation of the right pulmonary artery (RPA) anastomosis with homologous pericardial patch was performed. He was discharged from hospital with the advice to continue aspirin $75 \mathrm{mg}$ and warfarin $2 \mathrm{mg}$ orally daily. The child returned 1 year later with symptoms of breathlessness, increased cyanosis and hemoptysis. He had signs of venous congestion with normal sinus rhythm and heart rate of 100/ minutes in ECG. The International Normalized Ratio (INR) was 2.5 and prothrombin time of 35 seconds. He was suspected of thrombus in the TCPC circuit by transthoracic echo.

The child was scheduled for revision of the conduit. Intraoperative TEE (iE33 X-matrix machine, X7-2t 3D probe from Philips, Bothell, Wa, USA) confirmed a large thrombus at the superior aspect of the Fontan conduit and RPA anastomosis site (Fig. 1). A focal complete thrombotic occlusion of the circuit below the RPA for a length of approximately $3 \mathrm{~cm}$ was observed. A slight modification of the upper esophageal aortic arch short axis (SAX)

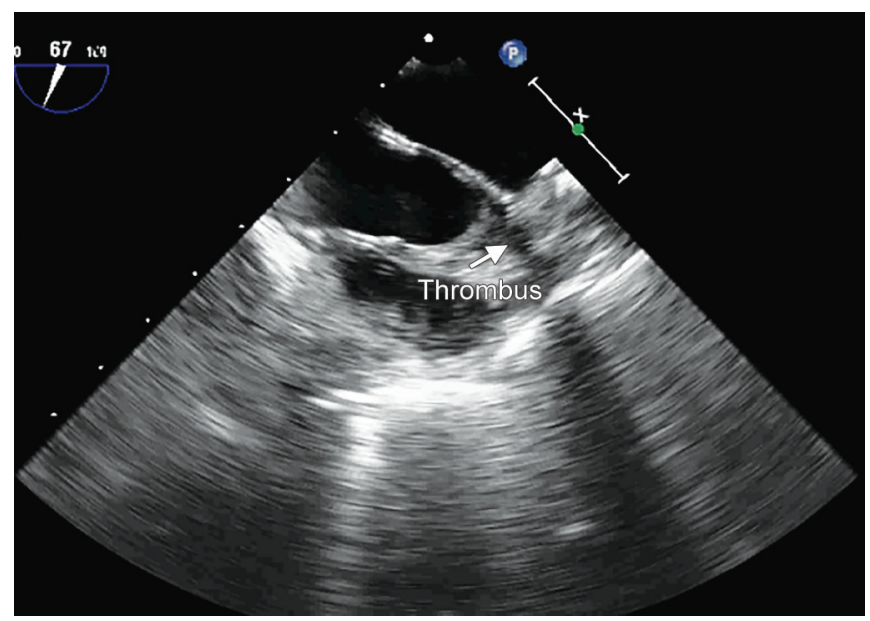

Fig. 1: Transesophageal echocardiography upper esophageal view showing thrombus (arrow) at the superior aspect of the Fontan conduit and RPA anastomosis site 


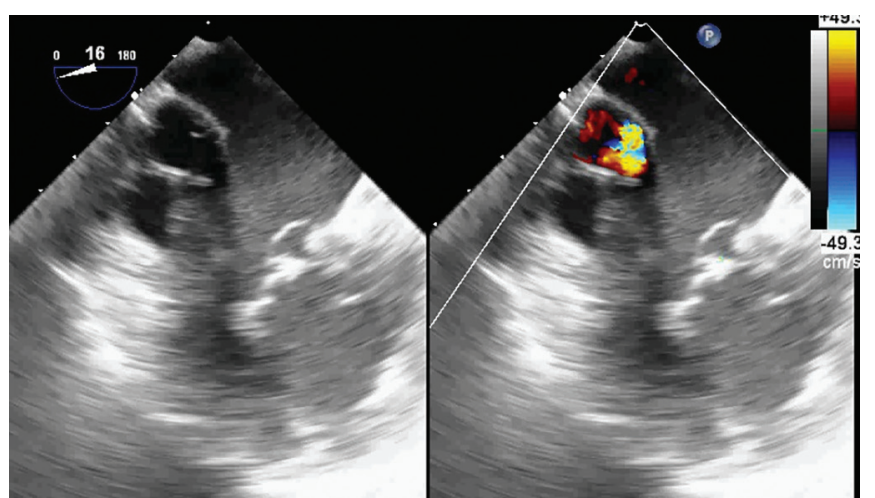

Fig. 2: Transesophageal echocardiography color Doppler showing turbulence in the Fontan conduit

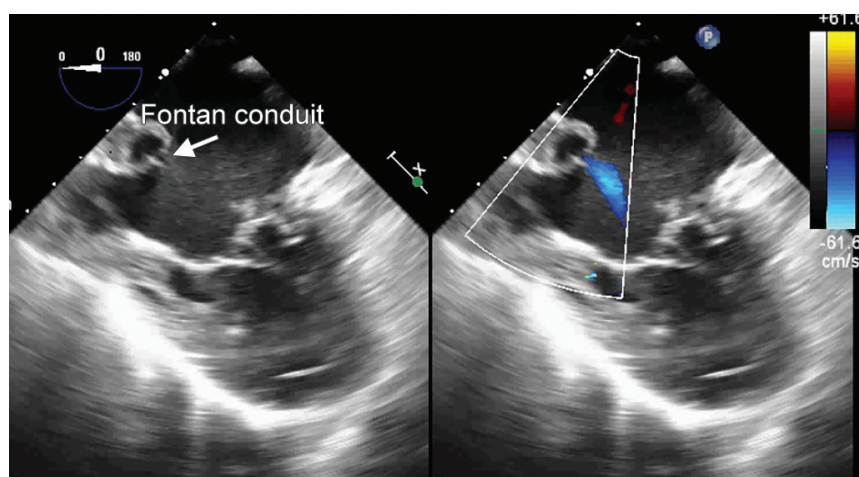

Fig. 3: Transesophageal echocardiography with color compare showing the patent fenestration of the Fontan conduit

view showed stenosis of the anastomotic site at the RPA along with the thrombus (Fig. 1). On color Doppler imaging, there was turbulence in the flow through the upper end of the circuit and the small flow was being obstructed by the thrombus (Fig. 2). A peak gradient of $3 \mathrm{~mm} \mathrm{Hg}$ was seen on continuous-wave Doppler echo at the anastomotic site. The inferior vena cava (IVC) end of the circuit was patent and flow across the fenestration could also be seen (Fig. 3). Three-dimensional (3D) TEE further helped in clearly delineating the thrombus and a well-circumscribed echogenic mass with clear borders was imaged in the RPA (Fig. 4). This guided the surgeon for exact location of thrombus intraoperatively.

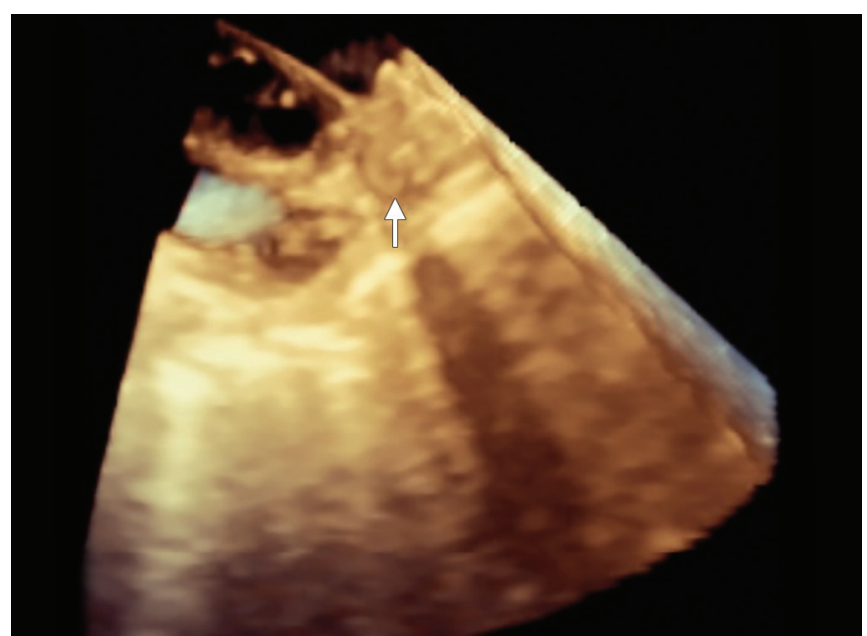

Fig. 4: Three-dimensional TEE showing the precise location of thrombus in the Fontan conduit

During surgery, a laminar thrombus was found adherent to the RPA. Surgical clot removal and revision of the Fontan circuit was performed. The successful complete removal of thrombus and free flow of blood in the Fontan circuit was visualized in TEE (Fig. 5).

\section{DISCUSSION}

Thrombus formation can occur in the atrium, pulmonary arteries and IVC after Fontan operation. ${ }^{1}$ Diagnosis of thrombosis usually occurs after the development of symptoms of pulmonary embolus and signs of systemic venous congestion. The location of the thrombus in the posterior structures, such as the caval atriopulmonary anastomosis, which is relatively inaccessible to imaging by transthoracic echocardiographic examination demonstrates the utility of the transesophageal technique ${ }^{2}$ but the disadvantage of TEE is that both the systemic and pulmonary veins may be imaged insufficiently. ${ }^{3}$ In our case, intraoperative TEE images were obtained after repeated modifications and sweeping of the structures with the probe. The midesophageal four-chamber view allowed us for the evaluation of the cavopulmonary connections

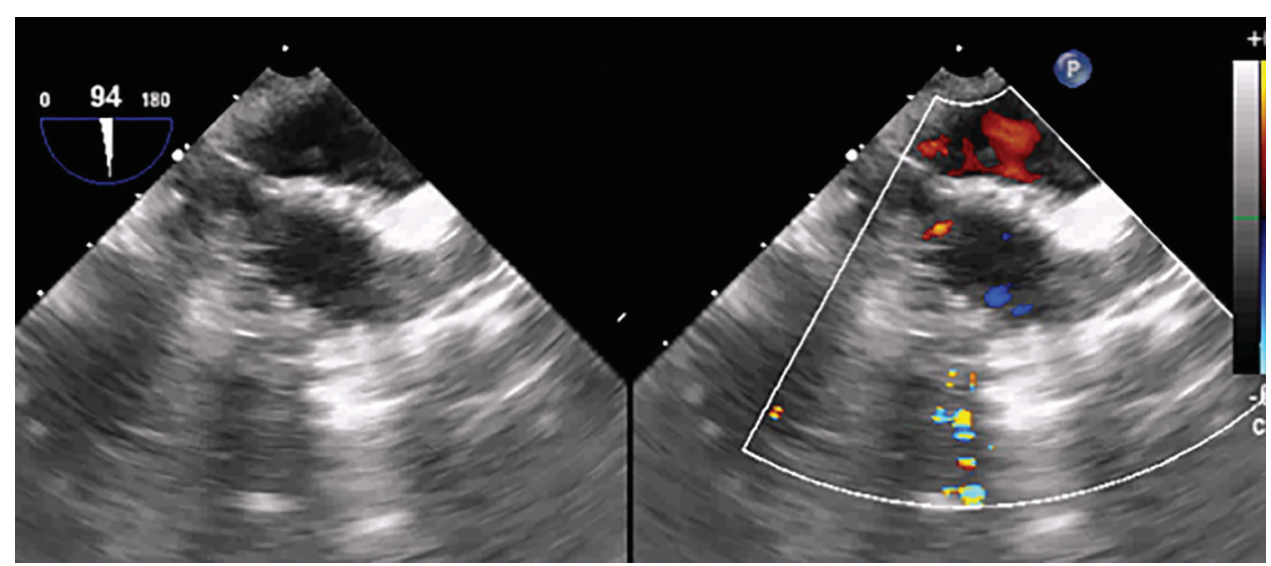

Fig. 5: Transesophageal echocardiography midesophageal bicaval view color compare showing the conduit in right atrium with a patent Fontan circuit without obstruction to blood flow 
and in demonstrating flow across the fenestration. A patent IVC-pulmonary artery (PA) communication was seen just to the right side of the right atrium by turning the probe to the right and withdrawal of the probe allowed for visualization of the patent IVC-PA connection. The upper esophageal aortic arch SAX view was particularly useful for examining the anastomosis between the conduit and RPA. Slight rotation of the echocardiography probe, toward the right, at the same level, enabled to image the thrombus in the circuit. Color Doppler was used to detect the turbulence in the blood flow (Fig. 2). During 3D TEE, the conduit was imaged from both zero and $90^{\circ}$ crystal rotation which facilitated in the diagnosis of the thrombus. Three-dimensional echocardiography can enhance the ability to detect or exclude thrombi within the Fontan conduit in comparison to two-dimensional (2D) TEE. The ability of 3D TEE to crop and manipulate the image, during both real-time imaging and off-line analysis helped us in identifying the exact location of the thrombus in the circuit and in planning the surgical approach. ${ }^{4}$ Our experience reveals that the walls of the Fontan conduit/thrombus are best seen when the gain set is very low. In our case, in spite of the use of autologous pericardium for augmentation and proper anticoagulation therapy, the stenosis at the RPA anastomosis was thought to be responsible for thrombus formation. As
Fontan circulation has low blood flow velocity, a state of passive stasis might develop in the conduit due to anastomotic site stricture, thus, hastening the formation of a clot in the TCPC circuit which can contribute to the symptoms.

We conclude that TEE has a beneficial role during TCPC surgery and should be carried out whenever possible. It is a sensitive technique for detecting thrombi and, in our case, was diagnostic of pulmonary artery thrombosis. In future, 3D TEE can have an important role in monitoring of thrombolytic therapy of Fontan circuit by providing both anatomic and physiologic evidence of resolution of thrombus.

\section{REFERENCES}

1. Jacobs ML, Pourmoghadam KK. Thromboembolism and the role of anticoagulation in the Fontan patient. Pediatr Cardiol 2007;28(6):457-464.

2. Balling G, Vogt M, Kaemmerer H, Eicken A, Meisner H, Hess J. Intracardiac thrombus formation after the Fontan operation. J Thorac Cardiovasc Surg 2000;119(4 Pt 1):745-752.

3. Gologorsky E, Gologorsky A, Rosenkranz E. An adult patient with Fontan physiology: a TEE perspective. Anesthesiol Res Pract 2012;2012(Article ID 475015):5.

4. Mart CR. Three-dimensional echocardiographic evaluation of the Fontan conduit for thrombus. Echocardiography 2012; 29(3):363-368. 